\title{
Frequency Tunability and Spectral Control in Terahertz Quantum Cascade Lasers With Phase-Adjusted Finite-Defect-Site Photonic Lattices
}

\author{
Iman Kundu, Paul Dean, Alexander Valavanis, Lianhe Li, Yingjun Han, \\ Edmund H. Linfield, and Alexander Giles Davies
}

\begin{abstract}
We report on the effect of finite-defect-site photonic lattices (PLs) on the spectral emission of terahertz frequency quantum cascade lasers, both theoretically and experimentally. A central $\pi$-phase adjusted defect incorporated in the PL is shown to favor emission selectively within the photonic bandgap. The effect of the duty cycle and the longitudinal position of such PLs is investigated, and used to demonstrate three distinct spectral behaviors: single-mode emission from devices in the range $2.2-5 \mathrm{THz}$, with a side-mode suppression ratio of $40 \mathrm{~dB}$ and exhibiting continuous frequency tuning over $>8 \mathrm{GHz}$; discrete tuning between two engineered emission modes separated by $\sim 40 \mathrm{GHz}$; and multiple-mode emission with an engineered frequency spacing between emission lines.
\end{abstract}

Index Terms-Gratings, laser tuning, semiconductor lasers, terahertz (THz), quantum cascade lasers (QCLs).

\section{INTRODUCTION}

$\mathbf{T}$ ERAHERTZ (THz) frequency quantum cascade lasers (QCLs) are unipolar semiconductor sources of $\mathrm{THz}$ radiation in which photons are emitted through electron intersubband transitions [1]. These compact semiconductor lasers have seen a remarkable development over the last decade and now operate over a frequency range of $\sim 1.2-5.6 \mathrm{THz}$ [2], [3], at a maximum temperature of $\sim 200 \mathrm{~K}$ [4], and can emit over $1 \mathrm{~W}$ of peak output power [5], [6]. THz QCLs commonly employ plasmonic FabryPérot (FP) waveguides to confine the radiation within the active material, and typically emit multiple longitudinal FP modes, whose frequency spacing is determined by the cavity length [7]. However, a stable single-mode frequency-tunable $\mathrm{THz}$ source is highly desirable for a number of applications including trace gas

Manuscript received January 9, 2017; revised March 28, 2017; accepted May 17, 2017. Date of publication June 14, 2017; date of current version June 29 , 2017.This work was supported in part by the Engineering and Physical Sciences Research Council, U.K. (COTS Programme EP/J017671/1, and EP/J002356/1) and in part by the Royal Society and Wolfson Foundation. Research data associated with this paper are openly available from The University of Leeds data repository: http://doi.org/10.5518/146. (Corresponding author: Iman Kundu.)

The authors are with the Institute of Microwaves and Photonics, School of Electronic and Electrical Engineering, The University of Leeds, Leeds LS2 9JT, U.K. (e-mail: I.Kundu@ leeds.ac.uk; P.Dean@leeds.ac.uk; A.Valavanis@leeds. ac.uk; L.H.Li@leeds.ac.uk; Y.Han@leeds.ac.uk; E.H.Linfield@leeds.ac.uk; G.Davies@leeds.ac.uk).

Color versions of one or more of the figures in this paper are available online at http://ieeexplore.ieee.org.

Digital Object Identifier 10.1109/TTHZ.2017.2707800 detection [8], atmospheric sensing [9], heterodyne mixing [10], spectroscopy, imaging, and laser feedback interferometry [11].

Single-mode emission from $\mathrm{THz}$ QCLs has been demonstrated previously using distributed feedback (DFB) waveguides [12]-[15] and photonic crystals [16], [17]. Although electrical and thermal tuning of DFB THz QCLs over $\sim 5 \mathrm{GHz}$ has been reported (centered at $2.5 \mathrm{THz}$, and operating in continuous wave) [18], this tuning range has been extended further through gas condensation and controlled deposition of a dielectric material [19], microelectromechanical actuators [20], and coupled microcavity mirrors [21]. However, the development of DFB lasers can be limited by lithographic fabrication constraints, and any subwavelength variation in the DFB pitch can change the emission frequency [22]. Moreover, the fabrication of DFB QCLs by optical lithography requires the design and fabrication of appropriate photomasks, which is not ideal for rapid prototyping.

Single-mode emission in THz QCLs has also been demonstrated by patterning a finite defect site photonic lattice (PL) onto the laser ridge using focused ion beam (FIB) milling [23]. Unlike DFB QCLs where the lattice is patterned along the entire laser cavity, the lattices in PL-QCLs are patterned only over a section of the cavity. As such, feedback in DFB lasers is provided by radiation scattering off the lattice, whereas feedback in PL-QCLs is provided by the cleaved mirror facets. FIB milling allows the postprocessing of packaged or previously characterized devices, and is ideal for rapid prototyping. However, the side-mode suppression ratio (SMSR) achieved from such PL-QCLs was reported only to vary over the range $12-25 \mathrm{~dB}$, and the degree of frequency tuning observed from such devices was not reported [23]. Moreover, the periodic lattice used in the previous demonstration allows emission unpredictably either at the edges of the photonic stopband or at the Bragg frequency.

In this paper, the emission characteristics of PL-QCLs are simulated using transfer matrices to model the entire laser cavity including the PL. A strictly periodic lattice forms a photonic stopband centered at the Bragg frequency, where emission is favored at either edge of the stopband. We show that by incorporating a central $\pi$-phase adjusted defect in the PL, following similar design principles used in DFB lasers [13], [24], emission close to the Bragg frequency can be engineered. We report on the effect of the duty cycle and the longitudinal position of such PLs on the emission characteristics. Through optimizing the 
PL-QCL designs, we demonstrate the following three distinct spectral behaviors.

1) Single-mode emission with a high SMSR and exhibiting continuous frequency tuning through control of the driving current and heat sink temperature.

2) Discrete tuning between two engineered emission lines.

3) Multiple-mode emission with an engineered mode spacing.

The first of these designs would be suitable for gas sensing and spectroscopic applications [25], as well as self-mixing interferometry applied to materials analysis and three-dimensional imaging [26], where wide and continuous frequency tuning is desired. The second design would be suited to applications including dual-frequency imaging [27], where controlled emission at widely spaced frequencies is required. The third design provides a means to achieve a longitudinal mode spacing that is independent of the cavity length. Such design principles can be applied to more complex multicavity $\mathrm{THz}$ QCLs, such as coupled-cavity THz QCLs to optimize Vernier effect-based frequency tuning [28], [29].

Although we have recently demonstrated integration of PLs into coupled-cavity THz QCLs [29], here we present comprehensive simulations and experimental results from single-cavity $\mathrm{THz}$ QCLs, and present a design process through which greater spectral control is achieved.

This paper is arranged as follows. Section II describes the fabrication and experimental results from an unpatterned $\mathrm{THz}$ QCL. The modeling and design of PL-QCLs using transfer matrices is presented in Section III. Experimental results obtained from a range of PL-QCLs are presented in Section IV. Finally, a summary is presented in Section V.

\section{FABRICATION AND CHARACTERIZATION OF THZ QCLS With Single Plasmon WAVEGUIDE}

$\mathrm{THz}$ QCLs based on active region design reported in [30] were rescaled to operate at 3.4-3.6 THz, and were grown using molecular beam epitaxy. Growth started with a 250 -nm-thick GaAs buffer layer, followed by a 300-nm-thick $\mathrm{Al}_{50} \mathrm{Ga}_{50} \mathrm{As}$ etch stop layer grown on a semiinsulating GaAs substrate. A 700-nm-thick $n$-doped GaAs buried contact layer (doped with Si at $2 \times 10^{18} \mathrm{~cm}^{-3}$ ) was grown next. This was followed by 115 repetitions of the active-region layers grown in the $\mathrm{GaAs} / \mathrm{Al}_{0.15} \mathrm{Ga}_{0.85} \mathrm{As}$ material system. Finally, an 80-nm-thick $n$-doped GaAs layer forming the top contact (doped with $\mathrm{Si}$ at $5 \times 10^{18} \mathrm{~cm}^{-3}$ ) was grown. THz QCL devices were processed with surface plasmon ridge waveguides of width $150 \mu \mathrm{m}$ and thickness $13.8 \mu \mathrm{m}$, and individual lasers were cleaved and packaged. The device was mounted and characterized in a Janis ST-100 continuous-flow helium-cooled cryostat.

In the first instance, THz QCLs without any PL were characterized under pulsed driving conditions $(10-\mathrm{kHz}$ current pulses operating at $2 \%$ duty cycle and gated with a modulation envelope of $165 \mathrm{~Hz}$ ). A photoconductive Ge:Ga detector was used to record the $\mathrm{THz}$ power at a range of QCL heat-sink temperatures, $T_{\text {HS }}$. Fig. 1(a) shows the light-current/current density-voltage (LIV) of an exemplar 2-mm-long device, as a function of the drive current, $I_{\mathrm{QCL}}$. A threshold current density

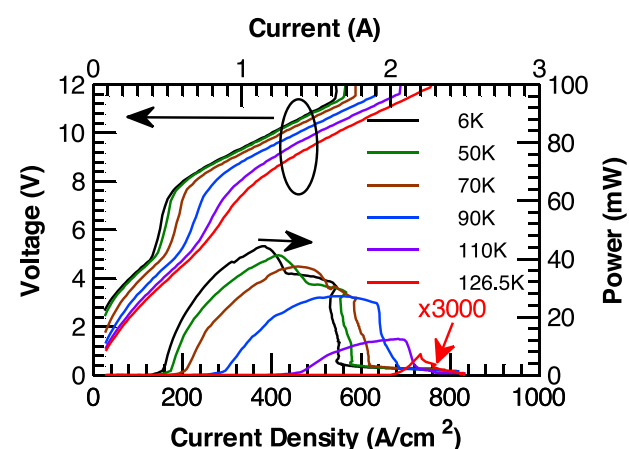

(a)

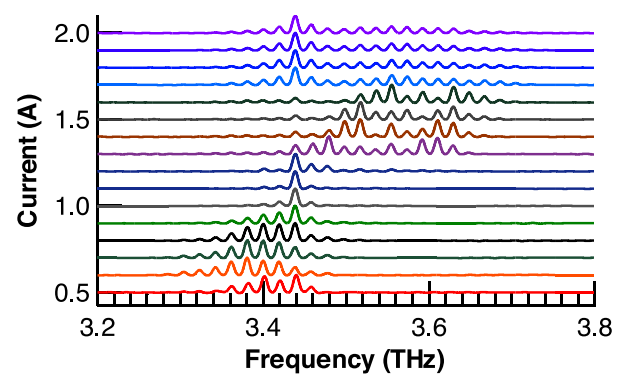

(b)

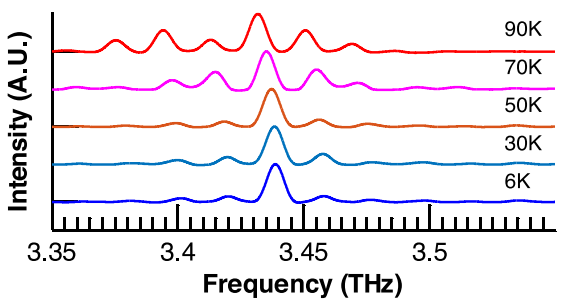

(c)

Fig. 1. LIV and emission spectra from a 2-mm-long unpatterned THz QCL. (a) LIV (peak THz power) at different $T_{\mathrm{HS}}$. (b) Spectra as a function of $I_{\mathrm{QCL}}$ at $T_{\mathrm{HS}}=6 \mathrm{~K}$. Blueshift of emission frequency is observed with an increase in $I_{\mathrm{QCL}}$. (c) Spectra at $I_{\mathrm{QCL}}=1.10 \mathrm{~A}$ and 6, 30, 50, 70 and $90 \mathrm{~K}$. Redshift of emission frequency is observed with an increase in $T_{\mathrm{HS}}$.

of $125 \mathrm{~A} / \mathrm{cm}^{2}$, a peak output power of $44.5 \mathrm{~mW}$ at $T_{\mathrm{HS}}=6 \mathrm{~K}$, and a maximum operating temperature of $\sim 126.8 \mathrm{~K}$ were determined. QCL emission spectra were also acquired as a function of $I_{\mathrm{QCL}}$ and $T_{\mathrm{HS}}$ [see Fig. 1(b) and (c)] using a Bruker IFS66 Fourier Transform Infrared spectrometer. Multiple-mode emission in the range 3.3-3.7 THz was observed, along with a blueshift in the emission frequencies with an increase in $I_{\mathrm{QCL}}$ [see Fig. 1(b)]. Furthermore, a redshift of the emission frequencies was observed with an increase in $T_{\mathrm{HS}}$, arising due to a shift in the gain spectrum of the active material [see Fig. 1(c)].

\section{DESIGN OF PL-QCLS}

Following the initial characterization of each device, PLs were designed for patterning onto the laser ridge using FIB milling. PL defect sites are formed by etching the gold cladding metal on top of the laser ridge and into the active region, which results in a change of the refractive index of the optical mode in the cavity. A periodic arrangement of these defect sites forms a plasmonic lattice. As such, to form a photonic stopband effectively, a PL with a large refractive index contrast $\left(\Delta n_{\mathrm{eff}}=n_{\mathrm{eff}, m}-n_{\mathrm{eff}, e}\right)$ between the metallized $\left(n_{\mathrm{eff}, m}\right)$ and the etched $\left(n_{\mathrm{eff}, e}\right)$ waveguide regions is desired. In order to 
(a)

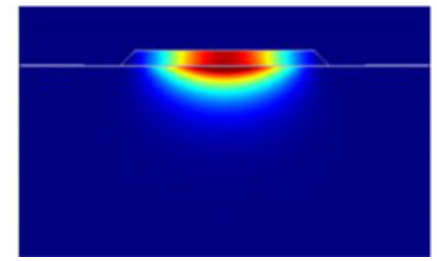

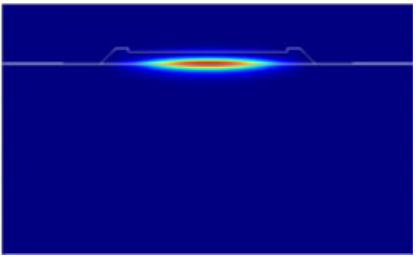

(b)
Fig. 2. Simulated optical mode intensity inside the THz QCL waveguide cross section of width $150 \mu \mathrm{m}$. The optical mode in (a) the metallized, and (b) the etched section $(130 \mu \mathrm{m})$, of the PL.

increase the refractive index contrast, a deep etch penetrating into the active material is used in this paper. Furthermore, in order to drive the PL section electrically, the etched PL defect sites did not extend the entire width of the ridge (unlike the PLQCLs reported in [23]). Instead, 130- $\mu$ m-wide etched sections were used in this paper (see Fig. 2).

The effective refractive index of the optical mode in the metallized sections was found to be $n_{\text {eff }, m}=3.67$, using a commercial finite element modeling software package (COMSOL Multiphysics) [see Fig. 2(a)]. A PL etch depth of $4 \mu \mathrm{m}$ was computed to provide an index contrast $\Delta n_{\text {eff }} \sim 0.6$ [see Fig. 2(b)]. The periodicity of the PL was calculated from the first-order Bragg wavelength $\left(\lambda_{\mathrm{BR}}\right)$. The length of the metallized $\left(L_{m}\right)$ and the etched $\left(L_{e}\right)$ sections of the PL are defined in terms of the PL duty cycle $(\sigma)$ using the relations

$$
\begin{aligned}
L_{m} & =\left(\lambda_{\mathrm{BR}} / 2\right) \sigma / n_{\mathrm{eff}, m} \\
L_{e} & =\left(\lambda_{\mathrm{BR}} / 2\right)(1-\sigma) / n_{\mathrm{eff}, e}
\end{aligned}
$$

The periodicity of the PL is defined as $\Lambda=L_{m}+L_{e}$.

\section{A. Modeling Transmission Characteristics of the PL}

In the first instance, the transmission characteristics of the PL were modeled using transfer matrices, as described in [31]. Frequency dependent complex refractive index was also included in the transfer matrix model. For simplicity, the gain spectrum of the active region (centered at $\sim 3.5 \mathrm{THz}$ ) was assumed to be invariant as a function of laser drive current. The matrix elements of a single defect site are defined as

$$
\begin{aligned}
& T_{11}=\frac{1}{t^{2}}\left[\exp \left(j \varphi_{+}\right)-r^{2} \exp \left(-j \varphi_{-}\right)\right] \\
& T_{12}=\frac{r}{t^{2}}\left[\exp \left(-j \varphi_{+}\right)-\exp \left(j \varphi_{-}\right)\right] \\
& T_{21}=\frac{r}{t^{2}}\left[\exp \left(j \varphi_{+}\right)-\exp \left(-j \varphi_{-}\right)\right] \\
& T_{22}=\frac{1}{t^{2}}\left[\exp \left(-j \varphi_{+}\right)-r^{2} \exp \left(j \varphi_{-}\right)\right]
\end{aligned}
$$

where $t$ and $r$ are the reflection and transmission coefficient calculated from the complex refractive indices at the PL aperture, and $\varphi_{+}=\beta_{m} L_{m}+\beta_{e} L_{e}$ and $\varphi_{-}=\beta_{m} L_{m}-\beta_{e} L_{e}$, where $\beta_{m}$ and $\beta_{e}$, are complex propagation constants in the metallized and etched sections of the PL, respectively. The transmission through a regular $\mathrm{PL}$ (without any central phase defect) consisting of a periodic cascade of $N=14$ repetitions of the unit PL were calculated using (3a). A central $\pi$-phase adjusted defect of

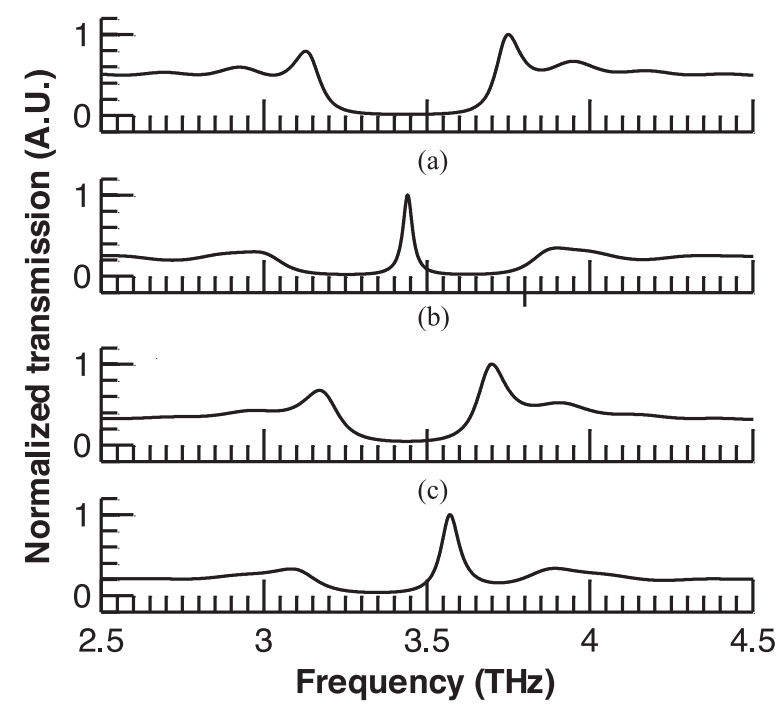

(d)

Fig. 3. Simulated normalized transmission through a PL designed at $f_{\mathrm{BR}}=$ $3.44 \mathrm{THz}\left(\lambda_{\mathrm{BR}}=87.209 \mu \mathrm{m}\right)$ with: (a) $\sigma=0.5$ without any central phase defect, (b) $\sigma=0.5$ with a central $\pi$-shifted phase defect, (c) $\sigma=0.75$ without any central phase defect, and (d) $\sigma=0.75$ with a central $\pi$-shifted phase defect.

length $L_{e}$ was also included, and the transmission through this phase adjusted PL was calculated using (3b),

$$
\begin{aligned}
T_{\mathrm{PL}, \mathrm{reg}} & =T^{N} \\
T_{\mathrm{PL}, \mathrm{ph} \text {.adj. }} & =T^{(N / 2)} \times T_{p i} \times T^{(N / 2)}
\end{aligned}
$$

Here, $T_{p i}$ is the transmission matrix of the central $\pi$ phase shift element of length $L_{e}$. Simulated transmission characteristics through a regular and a phase-adjusted PL with a Bragg frequency $f_{\mathrm{BR}}=3.44 \mathrm{THz}\left(\lambda_{\mathrm{BR}}=85.209 \mu \mathrm{m}\right)$ and $\sigma=0.50$ and 0.75 are shown in Fig. 3. Whereas a photonic stopband centered at $f_{\mathrm{BR}}$ is formed by the regular PL, for which emission is supported only at the edges of the stopband due to lower threshold gain, the phase-adjusted PL supports a transmission peak within this stopband. A slight asymmetry in the values of the transmission peaks at the lower and upper edges of the photonic stopband are due to the frequency dependent refractive index, and the difference between the Bragg frequency at $3.44 \mathrm{THz}$ and the peak gain at $3.5 \mathrm{THz}$. Furthermore, for a phase adjusted PL with $\sigma=0.50$ this transmission peak coincides with $f_{\mathrm{BR}}$ [see Fig. 3(a) and (b)]. Whereas increasing the duty cycle to $\sigma=0.75$ results in a similar photonic stopband from a regular $\mathrm{PL}$, the transmission peak blueshifts in frequency to $3.57 \mathrm{THz}$ in a phase adjusted PL [see Fig. 3(c) and (d)]. In order to reduce the FIB etching time of the PL and reduce the associated ion beam drift, a PL duty cycle of $\sigma=0.75$ was selected for this study. The coupling strength of the PL was calculated to be $\sim 2.6$ in this case [32].

\section{B. Modeling Transmission Characteristics of the Laser Cavity and the $P L$}

Following calculation of the transmission characteristics of the PL, the characteristics of the QCL cavity incorporating the PL were modeled using similar transfer matrices. The model comprises a front cavity section of length $L_{F}$, the PL section 


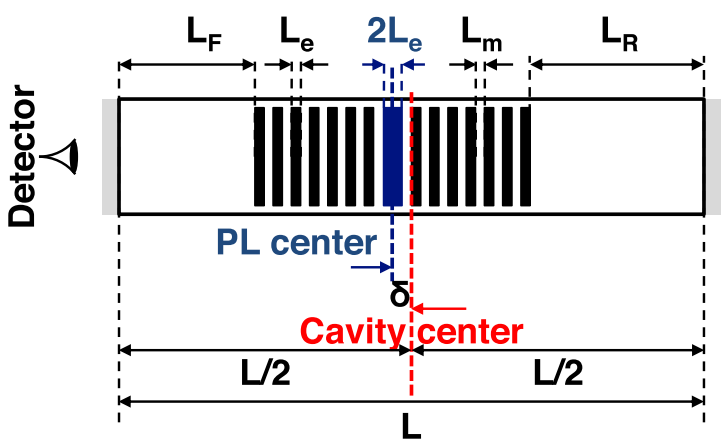

(a)

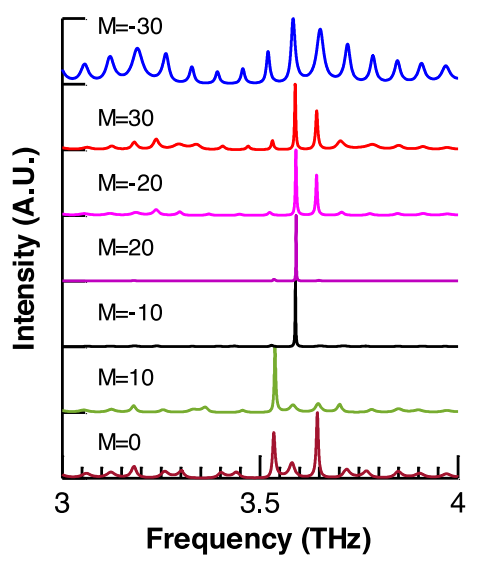

(b)

Fig. 4. (a) Schematic diagram of the THz QCL with a central $\pi$-phase-adjusted PL. The center of the PL is offset from the center of the QCL cavity by $\delta$. (b) Simulated normalized transmission through a 2-mm-long QCL with a central $\pi$-phase-adjusted PL as function of displacement factor $(M)$.

of length $\left(N \Lambda+L_{e}\right)$, and a rear cavity section of length $L_{R}$. A schematic diagram of the PL-QCL is shown in Fig. 4(a). The transmission of this cavity is calculated using

$$
T_{\mathrm{QCL}}=T_{F} \times T_{\mathrm{PL}, \mathrm{ph} . \mathrm{adj} .} \times T_{R}
$$

where $T_{F}$ and $T_{R}$ are the transmission matrices of the front and rear cavities, comprising the air-cavity interface and propagation paths of length $L_{F}$ and $L_{R}$, respectively.

Each defect site of the PL results in a periodic spectral modulation. The periodicity of such spectral modulation depends on the longitudinal position of the defect. A periodic arrangement of the PL defects results in a summation of such spectral modulations [33], [34]. Whereas, PLs are designed to suppress most FP modes favoring transmission at specific frequencies [35], the inherent spectral modulation arising due to the PLs can also be engineered such that multiple transmission peaks are supported. As such, the longitudinal position of the PL along the laser stripe is also important, since variation of this position changes the phase matching condition between the scattered forward and backward waves from the individual PL defect sites, and the facet reflections. The emission characteristics of PL-QCLs can be engineered by varying not only the PL dimensions but also its longitudinal position in the cavity. To this end, in our model, the center of the PL is displaced from the center of the cavity by a distance $\delta$ [see Fig. 4(a)]. For simplicity, we have defined $\delta=L_{F}-L_{R}$. The transmission

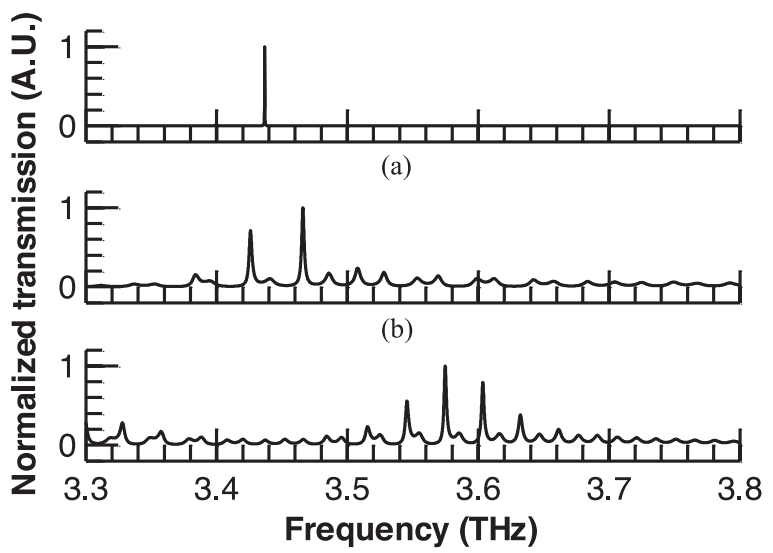

(c)

Fig. 5. Simulated normalized transmission from: (a) device 1 optimized for continuous tuning, (b) device 2 optimized for discrete tuning, and (c) device 3 optimized for multimode emission with an engineered mode spacing.

characteristics of a 2-mm-long cavity with a $\pi$-phase-adjusted $\mathrm{PL}\left(f_{\mathrm{BR}}=3.5 \mathrm{THz}, \lambda_{\mathrm{BR}}=85.714 \mu \mathrm{m}\right.$, and $\left.\sigma=0.75\right)$, simulated as a function of $\delta$, are shown in Fig. 4(b). For simplicity, $\delta$ is varied as $\pm M \lambda_{\mathrm{BR}} /\left(2 n_{\mathrm{eff}, m}\right)$, where we define a displacement factor $M=10,20,30$.

Whereas the transmission can be greatly enhanced at a specific frequency allowing single-mode emission with a high SMSR (for example when $M=-10,20$ ), varying the location of the PL can also favor emission at two distinct frequencies (for example when $M=0,-20,30)$. The inherent shift of the material gain as a function of $I_{\mathrm{QCL}}$ and $T_{\mathrm{HS}}$ can then be exploited to tune the emission between these two distinct frequencies, as demonstrated below. Interestingly, multiple-mode emission is also predicted when $M=-30$. Moreover, although the FP mode spacing for the 2-mm-long cavity is calculated to be $\sim 20 \mathrm{GHz}$, the mode spacing of the allowed modes in this case is $\sim 65 \mathrm{GHz}$. This phenomenon can be exploited to design QCLs with an engineered mode spacing, as also demonstrated below.

\section{Design of $P L-Q C L$}

The transfer matrix model described in the previous section was used to design three PL-QCLs to demonstrate three distinct spectral behaviors: continuous tuning of a single mode with high SMSR; discrete tuning between two engineered emission lines; and multiple-mode emission with an engineered mode spacing.

1) Device 1: In the first instance, the same 2-mm-long QCL shown in Fig. 1 was used to incorporate a PL with $\Lambda=12.93 \mu \mathrm{m}, L_{e}=3.11 \mu \mathrm{m}, f_{\mathrm{BR}}=3.44 \mathrm{THz}$, and $\delta=$ $-230 \mu \mathrm{m}$. In this design, the displacement of the PL $\delta$ is adjusted so that the transmission peak of the PL-QCL cavity coincides with the Bragg frequency of the isolated PL [see Fig. 5(a)]. Single-mode emission at $\sim 3.437 \mathrm{THz}$ with an SMSR of $38 \mathrm{~dB}$ is therefore predicted, making this design ideal for demonstrating single-mode continuous frequency tuning.

2) Device 2: In order to demonstrate discrete tuning between engineered cavity modes, a second device $(1.76 \mathrm{~mm}$ long) was designed incorporating a PL with $\Lambda=12.52 \mu \mathrm{m}$, $L_{e}=3.44 \mu \mathrm{m}, f_{\mathrm{BR}}=3.367 \mathrm{THz}$, and $\delta=0 \mu \mathrm{m}$. This device was designed such that the cavity supports a dominant peak at 
$\sim 3.5 \mathrm{THz}$ and a secondary peak at $<3.5 \mathrm{THz}$. This arrangement, in conjunction with the inherent blueshift of the emission spectra as the drive current or operating temperature are varied [see Fig. 1(b) and (c)], enables discrete tuning between these modes. Discrete tuning over $\sim 40 \mathrm{GHz}$ with peak transmissions at 3.426 and $3.466 \mathrm{THz}$ are predicted for this device [see Fig. 5(b)].

3) Device 3: Finally, a 2.70-mm-long device was used for a PL-QCL with $\Lambda=11.99 \mu \mathrm{m}, L_{e}=1.79 \mu \mathrm{m}, f_{\mathrm{BR}}=3.60 \mathrm{THz}$, and $\delta=70 \mu \mathrm{m}$. This PL-QCL was designed to support multimode transmission in the frequency range $\sim 3.5-3.65 \mathrm{THz}$, corresponding to the broad emission range observed in the unpatterned device at current densities $J_{\mathrm{QCL}}>450 \mathrm{~A} / \mathrm{cm}^{2}$ [see Fig. 1(b)]. In this case, multimode transmission peaks at 3.546, 3.575, 3.604, and $3.633 \mathrm{THz}$ (each with an SMSR $<10 \mathrm{~dB})$ are predicted. Furthermore, although the FP mode spacing for the unpatterned 2.7 -mm-long cavity is $\sim 15 \mathrm{GHz}$, the mode spacing between the simulated transmission peaks of the PL-QCL is calculated to be $\sim 29 \mathrm{GHz}$ (almost twice the FP mode spacing of the unpatterned cavity). This design principle can be further optimized by selecting the PL to be a higher order Bragg lattice, and by varying $\delta$ to design PL-QCLs with mode spacing in multiples of the FP mode spacing of the unpatterned cavity.

\section{EXPERIMENTAL RESULTS}

A dual beam FIB system (FEI Nova 200 NanoLab) with a high-resolution field emission gun scanning electron microscope (SEM) was used to mill the PL patterns onto packaged and bonded THz QCL ridges. A beam current of $7 \mathrm{nA}$ and an accelerating voltage of $30 \mathrm{kV}$ was used to mill the gold cladding metal from the top of the QCL ridge and into the active region to a depth $4 \mu \mathrm{m}$.

\section{A. Device 1}

The same 2-mm-long THz QCL described in Section II was used to fabricate the PL-QCL device 1 described in Section III-C. The device was characterized in pulsed mode as described in Section II. After patterning of the PL, the device operated at a higher threshold current density of $129 \mathrm{~A} / \mathrm{cm}^{2}$ and with a lower peak output power of $34 \mathrm{~mW}$, at a heat sink temperature $\left(T_{\mathrm{HS}}\right)$ of $6 \mathrm{~K}$, and with a maximum operating temperature of $\sim 118 \mathrm{~K}$ [see Fig. 6(a)]. This slight degradation in performance is due to the additional losses introduced in the cavity due to the PL. Nonetheless, the central phase defect resulted in singlemode emission at $\sim 3.441 \mathrm{THz}$, close to $f_{\mathrm{BR}}=3.44 \mathrm{THz}$, with an SMSR of $40 \mathrm{~dB}$ at peak output power (at $T_{\mathrm{HS}}=8 \mathrm{~K}$ ) [see Fig. 6(b)]. Spectra were recorded as a function of $I_{\mathrm{QCL}}$ and $T_{\mathrm{HS}}$, and the frequency of the peak emission was calculated to ascertain the tuning performance of the device. A continuous blueshift of $\sim 2 \mathrm{GHz}$ was observed as a function of $I_{\mathrm{QCL}}$ only, and a continuous redshift of the emission frequency of $\sim-6 \mathrm{GHz}$ was recorded by varying $T_{\mathrm{HS}}$ [see Fig. 6(c)]. A cumulative tuning range of over $8 \mathrm{GHz}$ with an optical output power of $>1 \mathrm{~mW}$ was thereby obtained from this device, making such designs ideal for spectroscopic and gas sensing applications.

The frequency gradient of the continuous tuning was calculated to be $1.6,2.1,3.1$, and $3.4 \mathrm{GHz} / \mathrm{A}$ at $T_{\mathrm{HS}}=8,50$, 70, and $90 \mathrm{~K}$, respectively [see Fig. 6(b)]. The increase in the

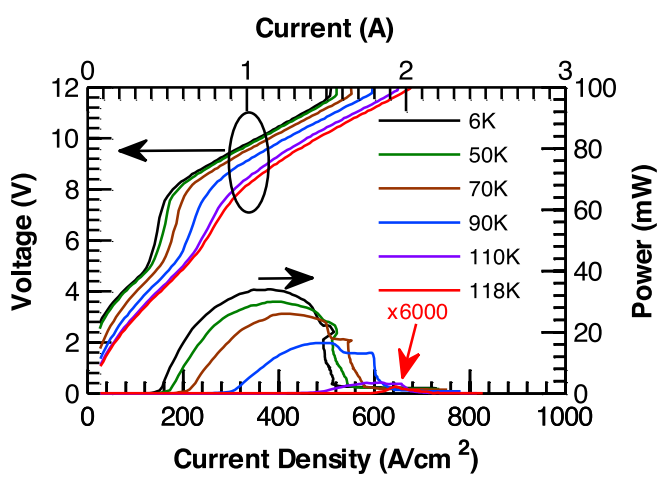

(a)

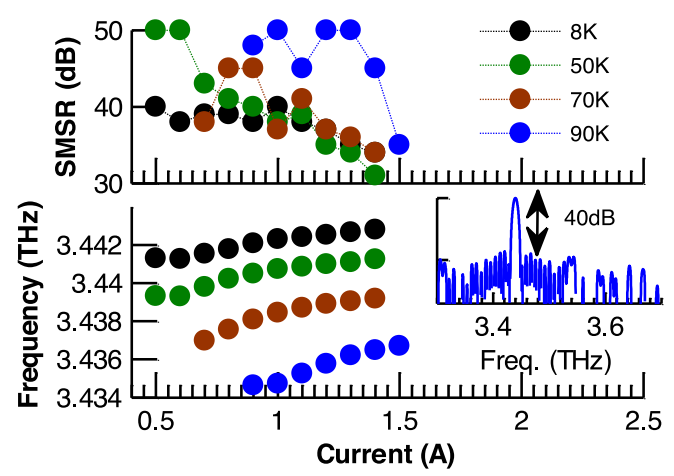

(b)

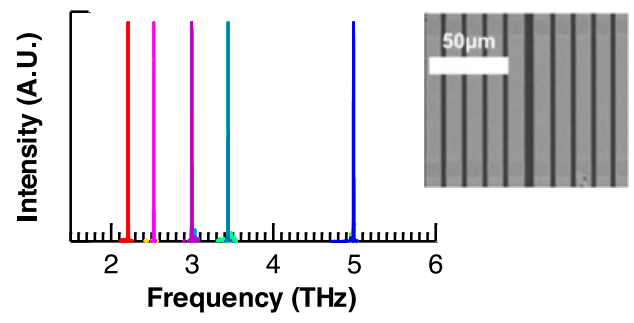

(c)

Fig. 6. Device 1. LIV and emission spectra from a 2-mm-long THz QCL after FIB patterning. (a) LIV at different $T_{\mathrm{HS}}$. (b) Frequency of peak emission and SMSR as a function of $I_{\mathrm{QCL}}$ at $T_{\mathrm{HS}}=8,50,70$, and $90 \mathrm{~K}$. Inset: Normalized emission spectra at peak output power and $T_{\mathrm{HS}}=8 \mathrm{~K}$ with an SMSR of $40 \mathrm{~dB}$. (c) Emission spectra from different THz QCLs with PLs spanning frequencies from 2.21-4.99 THz. Inset: SEM of patterned PL on device 1.

frequency gradient with an increase in $T_{\mathrm{HS}}$ is due to the change in refractive index at higher temperatures. As such, the frequency tunability can be further extended by adopting similar designs to THz QCLs with metal-metal waveguides, which operate at higher heat sink temperatures.

The SMSR was found to vary between $30-50 \mathrm{~dB}$ across the operating range of the device, with values $30-40 \mathrm{~dB}$ obtained after roll-off of the output power at $I_{\mathrm{QCL}}>\sim 1.2 \mathrm{~A}$ [see Fig. 6(b)]. Furthermore, a higher lattice temperature was found to result in an improvement in the SMSR due to an increase in the refractive index contrast. SMSR with values $>40 \mathrm{~dB}$ was obtained at peak output power when $T_{\mathrm{HS}}>50 \mathrm{~K}$. To demonstrate the applicability of this design principle for single-mode emission at a range of frequencies, PL-QCLs based on rescaled active regions reported in [6], [30], [36], and [37] were also designed and fabricated for emission at 4.99, 2.99, 2.53, and $2.21 \mathrm{THz}$, respectively [see Fig. 6(c)]. 


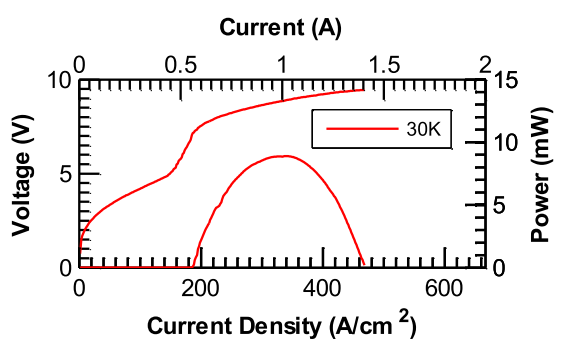

(a)

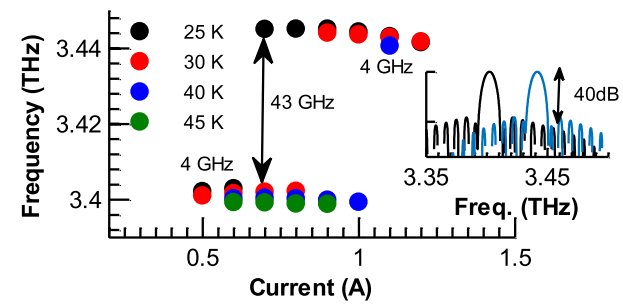

(c)

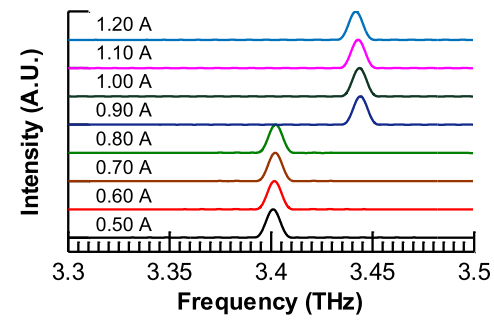

(b)

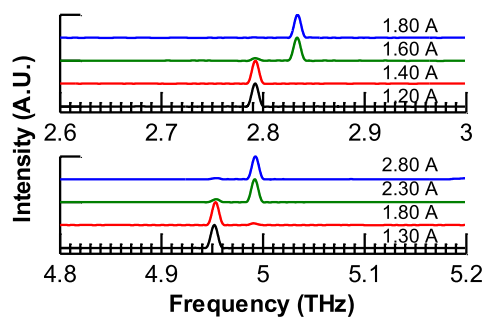

(d)

Fig. 7. Device 2 (continuous wave operation). (a) $\mathrm{LIV}$ at $T_{\mathrm{HS}}=30 \mathrm{~K}$. (b) Normalized emission spectra as a function of $I_{\mathrm{QCL}}$ at $T_{\mathrm{HS}}=30 \mathrm{~K}$. (c) Weighted averaged SPD at $T_{\mathrm{HS}}=25,30,40$, and $45 \mathrm{~K}$. A discrete mode hop of $43 \mathrm{GHz}$, and cumulative continuous tuning of $\sim 4 \mathrm{GHz}$ at both discrete modes is shown Inset: Normalized emission spectra at the two emission lines ( $T_{\mathrm{HS}}=30 \mathrm{~K}$ ) with an SMSR of $40 \mathrm{~dB}$. (d) Normalized emission spectra as a function of $I_{\mathrm{QCL}}$ at $T_{\mathrm{HS}}=8 \mathrm{~K}$ (pulsed mode operation) from two different THz QCLs based on similar design principle as device 2 .

\section{B. Device 2}

A QCL device with ridge length $1.76 \mathrm{~mm}$ was used to fabricate the PL-QCL device 2. In the first instance, LIV and spectra were recorded in continuous wave operation at $T_{\mathrm{HS}}=30 \mathrm{~K}$ [see Fig. 7(a) and (b)]. Single-mode emission at $3.402 \mathrm{THz}$, with an SMSR of $40 \mathrm{~dB}$, was observed with a drive current $I_{\mathrm{QCL}}=0.5 \mathrm{~A}$. With $I_{\mathrm{QCL}}=0.9 \mathrm{~A}$ the emission is seen to shift to a single mode at $3.445 \mathrm{THz}$. Whereas the emission frequencies are $\sim 23 \mathrm{GHz}$ lower than the simulated values, the discrete mode tuning of $\sim 43 \mathrm{GHz}$ agrees well with the simulated discrete tuning of $40 \mathrm{GHz}$ [see Fig. 5(b)]. In this case, an SMSR of $40 \mathrm{~dB}$ was observed across the operating range of the device. This value is consistent with that obtained in device 1 for heat sink temperatures $T_{\mathrm{HS}}>50 \mathrm{~K}$, suggesting that the higher lattice temperatures arising from continuous wave operation of this device facilitates the high SMSR. Furthermore, the mode at $\sim 3.44 \mathrm{THz}$ can be tuned continuously by $\sim-3.8 \mathrm{GHz}$ across the range $I_{\mathrm{QCL}}=0.5-1.2 \mathrm{~A}$. Spectra were similarly recorded at $T_{\mathrm{HS}}=25,40$, and $45 \mathrm{~K}$, and the tuning performance was calculated in each case from the weighted averaged spectral power density (SPD) as a function of $I_{\mathrm{QCL}}$ [see Fig. 7(c)]. Emission is observed to be predominantly at $\sim 3.40 \mathrm{THz}$ as $T_{\mathrm{HS}}$ is increased due to a redshift of the gain, and at $T_{\mathrm{HS}}=40 \mathrm{~K}$, no discrete tuning is observed. A cumulative continuous tuning of $\sim 4 \mathrm{GHz}$ was recorded for the two discrete modes by varying $T_{\mathrm{HS}}$ and $I_{\mathrm{QCL}}$. To demonstrate the reproducibility of this approach, $\mathrm{THz}$ QCLs based on rescaled active regions reported in [6], [36], were fabricated following similar design principles. Discrete emission at 2.792 and $2.833 \mathrm{THz}$, and at 4.952 and $4.992 \mathrm{THz}$ were observed as $I_{\mathrm{QCL}}$ was varied in both devices, respectively [see Fig. 7(d)].

\section{Device 3}

Following FIB patterning of the PL, the LIV, and spectral characteristics of device 3 were recorded in pulsed mode at $T_{\mathrm{HS}}=6 \mathrm{~K}$ [see Fig. 8(a) and (b)]. Single-mode emission at $3.492 \mathrm{THz}$ is observed for current densities $J_{\mathrm{QCL}}=197-400 \mathrm{~A} / \mathrm{cm}^{2}$, and multimode emission in the range $3.493-3.672 \mathrm{THz}$ is recorded at $J_{\mathrm{QCL}}>400 \mathrm{~A} / \mathrm{cm}^{2}$. This multiple-mode emission agrees well with the simulated transmission in the range $3.51-3.66 \mathrm{THz}$ for this device.

The evolution of the spectra from single-mode to multiplemode emission observed experimentally is explained by the blueshift of the gain of this QCL material with increasing bias.

Furthermore, we note that the mode spacing for this PL-QCL ( $\sim 28 \mathrm{GHz}$ ) is almost twice the FP longitudinal mode spacing for the unpatterned device $(\sim 15 \mathrm{GHz})$, and in close agreement with the simulated mode spacing of $\sim 29 \mathrm{GHz}$ [see Fig. 8(b)]. This is due to the first-order Bragg lattice designs used in this study for simplicity. The fact that the threshold current density and the dynamic range of operation for this device is similar to both the unpatterned device [see Fig. 1] and device 1 [see Fig. 6], demonstrates that the observed mode spacing results from the PL, and is not due to a selective operation of either the front or the rear section of the cavity. A similar spectral behavior was also recorded from another 2.93-mm-long $\mathrm{THz}$ QCL, which was patterned with a PL based on similar design principles [see Fig. 8(c)]. The longitudinal mode spacing of this device was observed to be $\sim 26 \mathrm{GHz}$, compared to the FP longitudinal mode spacing for the unpatterned device $(\sim 13.9 \mathrm{GHz})$.

This mode spacing can be further modified by using an aperiodic [38] or higher order lattice design. Indeed, a longitudinal mode spacing equal to three times that for the FP cavity has been simulated by using a second-order PL from a 2.7-mm-long cavity (FP mode spacing: $15 \mathrm{GHz}$ ) [see Fig. 8(d)].

Vernier effect based lasers have been demonstrated in visibleinfrared frequencies through more complex multisection lasers. Such lasers employ two longitudinal sampled-grating distributed Bragg reflectors (SG-DBRs), each of which results in a frequency comb [31]. However, adopting a similar design 


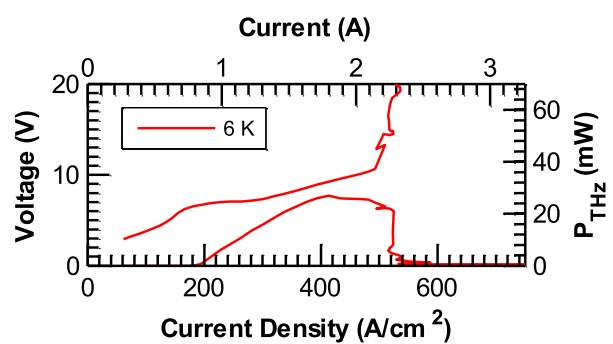

(a)

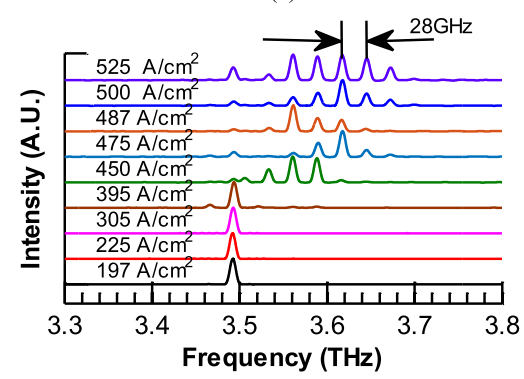

(b)

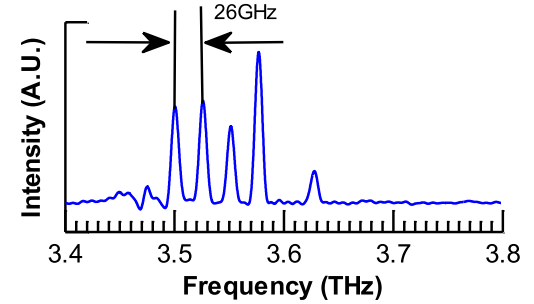

(c)

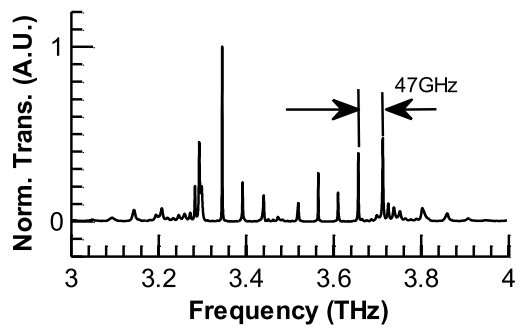

(d)

Fig. 8. Device 3. (a) LIV at $T_{\mathrm{HS}}=6 \mathrm{~K}$. (b) Normalized emission spectra at $T_{\mathrm{HS}}=6 \mathrm{~K}$ and at different $J_{\mathrm{QCL}}$. (c) Normalized emission spectra from a 2.93-mm-long THz QCL with a longitudinal mode spacing of $26 \mathrm{GHz}$. (d) Simulated normalized transmission from a 2.7-mm-long THz QCL with a second-order PL. Mode spacing of $47 \mathrm{GHz}$ has been simulated.

strategy for THz QCLs is challenging owing to the longer wavelength, meaning that each of the two SG-DBR cavities would need to extend over tens of millimeters. Instead, the approach to spectral control demonstrated here can be applied to optimize Vernier tuning using coupled cavity waveguides with integrated PLs [29].

\section{CONCLUSION}

In summary, THz QCLs incorporating finite defect site PLs have been developed with a central $\pi$-phase shift to favor transmission selectively within the photonic stopband. The PL design parameters and the longitudinal position of the PL within the cavity have been optimized to demonstrate three distinct spectral behaviors: single-mode emission with a high SMSR and exhibiting continuous frequency tuning through control of the driving current and heat sink temperature; discrete tuning between two engineered emission lines; and multiple-mode emission with an engineered mode spacing. In the first case, single-mode emission from devices in the range 2.2-5.0 THz, with an SMSR of 30-40 dB and exhibiting continuous frequency tuning over $\sim 8 \mathrm{GHz}$ has been demonstrated. Discrete tuning over $\sim 40 \mathrm{GHz}$ has also been reported. Finally, multiple-mode emission has been demonstrated with an engineered mode spacing of almost twice the longitudinal FP mode spacing of the unpatterned device. Such PL-QCLs can be used in THz applications such as gas spectroscopy, and laser feedback interferometry imaging, and to design frequency tunable THz QCLs with coupled cavities and integrated PLs.

\section{REFERENCES}

[1] R. Köhler et al., "Terahertz semiconductor-heterostructure laser," Nature, vol. 417, no. 6885 , pp. 156-159, May 2002.

[2] G. Scalari et al., "THz and sub-THz quantum cascade lasers," Laser Photon. Rev., vol. 3, no. 1-2, pp. 45-66, 2009.

[3] L. Li, I. Kundu, P. Dean, E. H. Linfield, and A. G. Davies, "High-power GaAs/AlGaAs quantum cascade lasers with emission in the frequency range 4.7-5.6 THz," in Proc. Int. Quantum Cascade Lasers School Workshop, Cambridge, U.K., 2016, pp. 87-88.
[4] S. Fathololoumi et al., "Terahertz quantum cascade lasers operating up to $\sim 200 \mathrm{~K}$ with optimized oscillator strength and improved injection tunneling," Opt. Exp., vol. 20, no. 4, pp. 3866-3876, Feb. 2012.

[5] M. Brandstetter et al., "High power terahertz quantum cascade lasers with symmetric wafer bonded active regions," Appl. Phys. Lett., vol. 103, no. 17, 2013, Art. no. 171113.

[6] L. Li et al., "Terahertz quantum cascade lasers with $>1$ W output powers," Electron. Lett., vol. 50, no. 4, pp. 309-311, Feb. 2014.

[7] B. S. Williams, "Terahertz quantum-cascade lasers," Nature Photon., vol. 1, no. 9, pp. 517-525, Sep. 2007.

[8] H.-W. Hübers et al., "High-resolution gas phase spectroscopy with a distributed feedback terahertz quantum cascade laser," Appl. Phys. Lett., vol. 89, no. 6, 2006, Art. no. 061115.

[9] M. Tonouchi, "Cutting-edge terahertz technology," Nature Photon., vol. 1, no. 2, pp. 97-105, Feb. 2007.

[10] H. W. Hubers, "Terahertz heterodyne receivers," IEEE J. Sel. Topics Quantum Electron., vol. 14, no. 2, pp. 378-391, Apr. 2008.

[11] P. Dean et al., "Terahertz imaging through self-mixing in a quantum cascade laser," Opt. Lett., vol. 36, no. 13, pp. 2587-2589, Jul. 2011.

[12] L. Mahler et al., "High-performance operation of single-mode terahertz quantum cascade lasers with metallic gratings," Appl. Phys. Lett., vol. 87, no. 18, 2005, Art. no. 181101.

[13] S. Kumar et al., "Surface-emitting distributed feedback terahertz quantumcascade lasers in metal-metal waveguides," Opt. Exp., vol. 15, no. 1, pp. 113-128, Jan. 2007.

[14] M. I. Amanti, M. Fischer, G. Scalari, M. Beck, and J. Faist, "Lowdivergence single-mode terahertz quantum cascade laser," Nature Photon., vol. 3, no. 10, pp. 586-590, Oct. 2009.

[15] F. Castellano et al., "Distributed feedback terahertz frequency quantum cascade lasers with dual periodicity gratings," Appl. Phys. Lett., vol. 106, no. 1, 2015, Art. no. 011103.

[16] Y. Chassagneux et al., "Electrically pumped photonic-crystal terahertz lasers controlled by boundary conditions," Nature, vol. 457, no. 7226, pp. 174-178, Jan. 2009.

[17] Y. Chassagneux et al., "Graded photonic crystal terahertz quantum cascade lasers," Appl. Phys. Lett., vol. 96, no. 3, 2010, Art. no. 031104.

[18] H.-W. Hübers et al., "Molecular spectroscopy with terahertz quantum cascade lasers," J. Nanoelectron. Optoelectron., vol. 2, no. 1, pp. 101107, Apr. 2007.

[19] D. Turčinková, M. I. Amanti, F. Castellano, M. Beck, and J. Faist, "Continuous tuning of terahertz distributed feedback quantum cascade laser by gas condensation and dielectric deposition," Appl. Phys. Lett., vol. 102, no. 18,2013 , Art. no. 181113.

[20] N. Han et al., "Broadband all-electronically tunable MEMS terahertz quantum cascade lasers," Opt. Lett., vol. 39, no. 12, pp. 3480-3483, Jun. 2014. 
[21] F. Castellano et al., "Tuning a microcavity-coupled terahertz laser," Appl. Phys. Lett., vol. 107, no. 26, 2015, Art. no. 261108.

[22] D. G. Allen, T. Hargett, J. L. Reno, A. A. Zinn, and M. C. Wanke, "Index tuning for precise frequency selection of terahertz quantum cascade lasers," IEEE Photon. Technol. Lett., vol. 23, no. 1, pp. 30-32, Jan. 2011.

[23] S. Chakraborty et al., "Spectral engineering of terahertz quantum cascade lasers using focused ion beam etched photonic lattices," Electron. Lett., vol. 42, no. 7, pp. 404-405, Mar. 2006.

[24] J. Carroll, J. Whiteaway, and D. Plumb, Distributed Feedback Semiconductor Lasers. London, U.K.: IEE, 1998.

[25] H. Richter et al., "4.7-THz local oscillator for the GREAT heterodyne spectrometer on SOFIA," IEEE Trans. THz Sci. Technol., vol. 5, no. 4, pp. 539-545, Jul. 2015.

[26] J. Keeley et al., "Three-dimensional terahertz imaging using sweptfrequency feedback interferometry with a quantum cascade laser," Opt. Lett., vol. 40, no. 6, pp. 994-997, Mar. 2015.

[27] P. Dean et al., "Dual-frequency imaging using an electrically tunable terahertz quantum cascade laser," Opt. Exp., vol. 17, no. 23, pp. 2063120641, Nov. 2009.

[28] I. Kundu et al., "Discrete Vernier tuning in terahertz quantum cascade lasers using coupled cavities," Opt. Exp., vol. 22, no. 13, pp. 16595$16605,2014$.

[29] I. Kundu et al., "Quasi-continuous frequency tunable terahertz quantum cascade lasers with coupled cavity and integrated photonic lattice," Opt. Exp., vol. 25, no. 1, pp. 486-496, Jan. 2017.

[30] M. Wienold et al., "Low-voltage terahertz quantum-cascade lasers based on LO-phonon-assisted interminiband transitions," Electron. Lett., vol. 45, no. 20, pp. 1030-1031, Sep. 2009.

[31] L. A. Coldren, S. W. Corzine, and M. L. Masanovic, Diode Lasers and Photonic Integrated Circuits, 2nd ed. Hoboken, NJ, USA: Wiley, 2012.

[32] H. Kogelnik and C. V. Shank, "Coupled-wave theory of distributed feedback lasers," J. Appl. Phys., vol. 43, no. 5, pp. 2327-2335, 1972.

[33] J. S. Young, D. A. Kozlowski, J. M. C. England, and R. G. S. Plumb, "Spectral perturbation and mode suppression in $1.3 \mu \mathrm{m}$ Fabry-Perot lasers," Electron. Lett., vol. 31, no. 4, pp. 290-291, Feb. 1995.

[34] D. A. Kozlowski, J. S. Young, J. M. C. England, and R. G. S. Plumb, "Singlemode $1.3 \mu \mathrm{m}$ Fabry-Perot lasers by mode suppression," Electron. Lett., vol. 31, no. 8, pp. 648-650, Apr. 1995.

[35] J. S. Foresi et al., "Photonic-bandgap microcavities in optical waveguides," Nature, vol. 390, no. 6656, pp. 143-145, Nov. 1997.

[36] S. Barbieri et al., "2.9 THz quantum cascade lasers operating up to $70 \mathrm{~K}$ in continuous wave," Appl. Phys. Lett., vol. 85, no. 10, pp. 1674-1676, 2004.

[37] C Worrall et al., "Continuous wave operation of a superlattice quantum cascade laser emitting at 2 THz," Opt. Exp., vol. 14, no. 1, pp. 171-181, Jan. 2006.

[38] S. Chakraborty et al., "Discrete mode tuning in terahertz quantum cascade lasers," Opt. Exp., vol. 20, no. 26, pp. B306-B314, Dec. 2012.

Iman Kundu received the B.Tech degree in electronics and communication engineering from the West Bengal University of Technology, Kolkata, India, in 2007, and the M.Sc. (Eng.) degree in nanotechnology and advanced electronic devices and Ph.D. degree in electronic and electrical engineering from The University of Leeds, Leeds, U.K., in 2010 and 2014, respectively.

From 2008 to 2009, he was an Assistant Systems Engineer with the Tata Consultancy Services, India, working on software development. Since 2014, he has been a Postdoctoral Research Fellow with The University of Leeds, Leeds, U.K. His research interest includes the fabrication and development of novel waveguides for THz QCLs, laser dynamic in coupled waveguides, fabrication of microstructures on QCL waveguides, and THz photonics-in-a-chip technology.

Paul Dean received the M.Phys. (Hons.) degree in physics and Ph.D. degree in laser physics from the University of Manchester, Manchester, U.K., in 2001 and 2005, respectively.

In 2005, he became a Postdoctoral Research Associate with the Institute of Microwaves and Photonics, School of Electronic and Electrical Engineering, The University of Leeds, Leeds, U.K., and as a University Research Fellow in 2011. His research interests include terahertz optoelectronics, quantum cascade lasers, and terahertz imaging techniques.
Alexander Valavanis received the M.Eng. (Hons.) degree in electronic engineering from the University of York, York, U.K., in 2004, and the Ph.D. degree in electronic and electrical engineering from The University of Leeds, Leeds, U.K., in 2009.

From 2004 to 2005, he was an instrumentation engineer with STFC Daresbury Laboratories, Warrington, U.K., and from 2009 to 2016 he was a Research Fellow with The University of Leeds. He is currently a University Academic Fellow (tenure track) in terahertz instrumentation with The University of Leeds. His research interests include terahertz instrumentation, quantum cascade lasers, silicon photonics, and computational methods for quantum electronics.

Dr. Valavanis is a member of the Institution of Engineering and Technology.

Lianhe Li received the Ph.D. degree in microelectronics and solid-state electronics from the Institute of Semiconductors, Chinese Academy of Sciences, Beijing, China, in 2001.

From 2001 to 2003, he was with the Laboratoire de Photonique et desNanostructures, Centre National de la Recherche Scientifique, France. He then joined the École polytechnique fédérale de Lausanne, Lausanne, Switzerland, as a Scientific Collaborator, working on InAs quantum dots for lasers and superluminescent LEDs and single quantum dot devices. Since 2008, he has been with the School of Electronic and Electrical Engineering, The University of Leeds, Leeds, U.K. His current research interests include the molecular beam epitaxy growth and characterization of semiconductor materials and devices with a particular emphasis on midinfrared and terahertz-frequency generation and detection.

Yingjun Han received the B.E. degree in automatic control from the Northwestern Polytechnical University, Xian, China, in 1998, and the Ph.D. degree from the Institute of Physics, Chinese Academy of Sciences, Beijing, China, in 2003.

In 2003, he joined the Paul Drude Institute, Berlin, Germany. In 2006, he joined the Shanghai Institute of Microsystem and Information Technology, Chinese Academy of Sciences. Since 2012, he has been a Postdoctoral Research Fellow with the School of Electronic and Electrical Engineering, The University of Leeds, Leeds, U.K. His research interests include the terahertz frequency electronics and photonics, quantum cascade lasers and their applications.

Edmund H. Linfield received the B.A. (Hons.) degree in physics and M.A. degree from the University of Cambridge, Cambridge, U.K., in 1986 and 1991, respectively, and the Ph.D. degree in semiconductor physics from the Cavendish Laboratory, University of Cambridge, in 1991.

He continued his research as a Postdoctoral Researcher Associate with the Cavendish Laboratory, University of Cambridge, until 1997, when he was appointed as an Assistant Director of Research. He also became a Fellow of Gonville and Caius College, Cambridge. In 2004, he became the Chair of Terahertz Electronics with The University of Leeds, Leeds, U.K., where he is also the Director of Research with the School of Electronic and Electrical Engineering. His research interests include semiconductor growth and device fabrication, terahertz-frequency optics and electronics, and nanotechnology.

Prof. Linfield was the recipient of the IOP Faraday Medal and Prize, jointly with Prof. A. G. Davies, in 2014 and a Wolfson Research Merit award from the Royal Society in 2015. He also received a Dream Fellowship from the Engineering and Physical Sciences Research Council in 2011.

Alexander Giles Davies received the B.Sc. (Hons.) degree in chemical physics from the University of Bristol, Bristol, U.K., in 1987, and the Ph.D. degree from the University of Cambridge, Cambridge, U.K., in 1991.

In 1991, he joined the University of New South Wales, Sydney, NSW, Australia, supported by an Australian Research Council Fellowship, before returning to the Cavendish Laboratory, University of Cambridge, in 1995 as a Royal Society University Research Fellow, and subsequently as the Trevelyan Fellow of Selwyn College, Cambridge. Since 2002, he has been a Professor of electronic and photonic engineering with the School of Electronic and Electrical Engineering, The University of Leeds, Leeds, U.K., and is currently also ProDean for research and innovation on the Faculty of Engineering. His research interests include the optical and electronic properties of semiconductor devices, terahertz frequency electronics and photonics, and the exploitation of biological properties for nanostructure engineering.

Prof. Davies is a Fellow of the Royal Academy of Engineering and the Institute of Physics. He is both a Chartered Physicist and Engineer. He was the recipient of a Wolfson Research Merit Award from the Royal Society in 2011 and shared the Faraday Medal and Prize from the Institute of Physics in 2014. 